The purpose of this Brief Communications section is to present important research results of more limited scope than regular articles appearing in Physics of Fluids A. Submission of material of a peripheral or cursory nature is strongly discouraged. Brief Communications cannot exceed three printed pages in length, including space allowed for title, figures, tables, references, and

an abstract limited to about 100 words.

\title{
Taylor vortices between elliptical cylinders
}

\author{
Mark R. Schumack ${ }^{\text {a) }}$ and William W. Schultz \\ Department of Mechanical Engineering and Applied Mechanics, University of Michigan, Ann Arbor, \\ Michigan 48109 \\ John P. Boyd \\ Department of Atmospheric, Oceanic, and Space Science, University of Michigan, Ann Arbor, \\ Michigan 48109 \\ (Received 20 August 1991; accepted 10 June 1992) \\ Critical parameters (Reynolds number and wave number) signaling the onset of Taylor \\ vortices are calculated for the flow between "elliptical" cylinders. The spinning inner cylinder \\ is circular; the stationary outer cylinder is composed of two circular arcs and is similar \\ to an ellipse. It is shown that increasing ellipticity destabilizes the flow and increasing \\ eccentricity stabilizes the flow. The spectral element method is used to calculate the \\ base flow and to solve the linear stability problem.
}

In this Brief Communication, we describe the linear stability of flow between a circular inner cylinder spinning with an angular speed $\omega$ and a stationary outer "elliptical" cylinder. Both cylinders are considered infinitely long. As seen in Fig. 1, the outer cylinder is not actually an ellipse, but an oblong shape constructed from two circular arcs of equal size. The use of "elliptical" to describe such a geometry is common in lubrication literature. For small clearances between the cylinders, this geometry resembles that of an elliptical journal bearing (actual bearings have finite length and are often fitted with lubricant inlet and outlet flow ports). Pinkus ${ }^{1,2}$ and Kumar et al. ${ }^{3}$ have studied torque and load characteristics based on solution of the lubrication equation. ${ }^{4}$ We discuss here the flow instability characterized by the appearance of Taylor vortices for the constrained location of the cylinder centers. Another type of bearing instability known as "shaft whirl" or "oil whip" arises from interaction between fluid forces and shaft translation and is discussed in Ref. 5. The increased shaft stability of an elliptical journal bearing makes it attractive for high-speed applications, such as in turbines or rotary compressors. ${ }^{6}$

The Taylor-Couette stability problem for flow between concentric circular cylinders has been extensively studied (see the review article by DiPrima and Swinney ${ }^{7}$ ). This flow lends itselr to analysis because the base flow is one dimensional and the exchange-of-stabilities principle can be used. When the cylinders are eccentrically placed and/ or of arbitrary shape, the base flow is Reynolds number dependent and exchange-of-stabilities cannot be assumed, substantially complicating the analysis. DiPrima and Stuart ${ }^{8}$ performed a perturbation analysis for eccentric circular cylinders assuming small clearance and eccentricity.
They showed that increasing eccentricity stabilizes the flow. Subsequent papers by DiPrima and Stuart ${ }^{9}$ and Eagles et al. ${ }^{10}$ investigated the nonlinear characteristics of Taylor vortices between eccentric circular cylinders. Oikawa et al. ${ }^{11}$ numerically investigated the linear stability of the flow between eccentric circular cylinders without the restrictions of small eccentricity or clearance. Oikawa's results show good agreement with the DiPrima and Stuart analysis for small eccentricity and clearance. They also agree well with experiments by Vohr ${ }^{12}$ and Karasudani. ${ }^{13}$

There are no analytical or numerical studies of the stability of flows between noncircular cylinders. Lewis ${ }^{14}$ solved for the base flow between a rotating circular inner cylinder and fixed square outer cylinder using finite differences. Snyder, ${ }^{15}$ Mullin, ${ }^{16}$ and Terada and Hattor ${ }^{17}$ experimentally studied flow stability for a variety of inner/outer noncircular cylinder arrangements and characterized the bifurcation to Taylor vortices for the various geometries.

The two nondimensional parameters describing the concentric elliptical geometry are the ellipticity ratio, $e^{*}=e / d$, and the minimum gap radius ratio, $\eta=R_{i} /\left(R_{i}+d\right)$, where $e$ is the distance between the inner cylinder center and the center of the circle describing either lobe of the outer cylinder, $d$ is the minimum gap width, and $R_{i}$ is the inner cylinder radius (see Fig. 1 ).

We use the spectral element method ${ }^{5,18-20}$ to solve the base flow and linear stability problems. For the twodimensional base flow, we solve the incompressible Navier-Stokes equations in a Cartesian coordinate system:

$$
\begin{aligned}
& \operatorname{Re}\left(\frac{\partial \mathbf{u}}{\partial t}+\frac{1}{2}(\mathbf{u} \cdot \nabla \mathbf{u}+\nabla \cdot \mathbf{u u})\right)=\nabla^{2} \mathbf{u}-\operatorname{Re} \nabla p, \\
& \nabla \cdot \mathbf{u}=0 .
\end{aligned}
$$




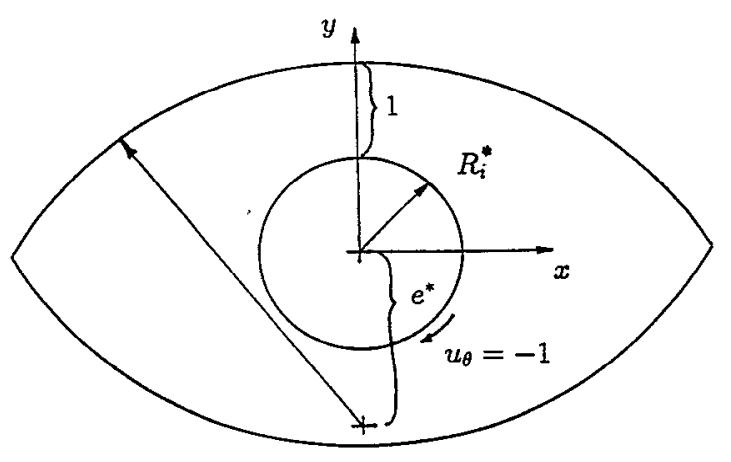

FIG. 1. Elliptical geometry. All parameters shown are dimensionless.

The Reynolds number is $\operatorname{Re}=\omega R_{i} d / v$, where $v$ is the kinematic viscosity. The skew-symmetric form for the nonlinear terms is used to minimize aliasing errors. ${ }^{21}$ The unknowns within an element are approximated in a series of orthogonal polynomials:

$$
\mathbf{u}=\sum_{m=0}^{N} \sum_{n=0}^{N} \mathbf{u}_{m n} h_{m}(r) h_{n}(s)
$$

and

$$
p=\sum_{m=0}^{N-2} \sum_{n=0}^{N-2} p_{m n} L_{m}(r) L_{n}(s),
$$

where $h_{m}(r)$ is the Lagrangian interpolant of order $N$ constructed from Legendre polynomials, $L_{m}(r)$ is the Legendre polynomial of order $m, \mathbf{u}_{m n}$ is the velocity at the $m n$th grid point, $p_{m n}$ is the $m n$th spectral coefficient for pressure, and $r$ and $s$ arc the computational coordinates related to the Cartesian coordinates by

$$
\mathbf{x}=\sum_{m=0}^{N} \sum_{n=0}^{N} \mathbf{x}_{m n} h_{m}(r) h_{n}(s)
$$

The pressure approximation is two orders less than the velocity approximation to avoid spurious pressure modes. ${ }^{19}$ The formulation consists of multiplying (1) and (2) by appropriate test functions, integrating by parts, inserting the approximations ( 3 ) and (4), and performing GaussLobatto quadrature. ${ }^{18,22} \mathrm{We}$ use an implicit time-marching scheme that allows large time steps to rapidly find steadystate solutions. $^{5}$

For the stability analysis, we assume small perturbations of the form

$$
\left\{\begin{array}{l}
u \\
v \\
w \\
p
\end{array}\right\}(x, y, z, t)=\left\{\begin{array}{l}
U \\
V \\
0 \\
p
\end{array}\right\}(x, y)+e^{\sigma t}\left\{\begin{array}{l}
\hat{u} \cos k z \\
\hat{v} \cos k z \\
\hat{w} \sin k z \\
\hat{p} \cos k z
\end{array}\right\},
$$

where $k$ is the real wave number along the $z$ axis and $\sigma$ is (in general) a complex growth rate. Substituting (6) into the nonconservative form of the three-dimensional NavierStokes equations and linearizing yields

$$
\begin{aligned}
& \operatorname{Re}(\sigma \hat{\mathbf{u}}+\mathbf{U} \cdot \nabla \hat{\mathbf{u}}+\hat{\mathbf{u}} \cdot \nabla \mathbf{U})=-\operatorname{Re} \nabla \hat{p}+\nabla^{2} \hat{\mathbf{u}}-k^{2} \hat{\mathbf{u}}, \\
& \operatorname{Re}(\sigma \hat{w}+\mathbf{U} \cdot \nabla \hat{w})=\operatorname{Re} k \hat{p}+\nabla^{2} \hat{w}-k^{2} \hat{w}, \\
& \nabla \cdot \hat{\mathbf{u}}+k \hat{w}=0 .
\end{aligned}
$$

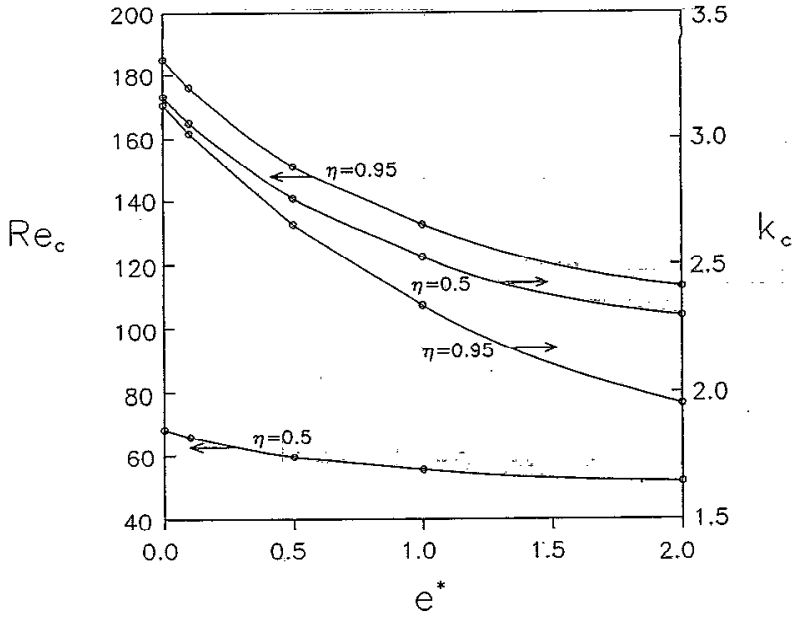

FIG. 2. Critical Reynolds number and wave number versus ellipticity for $\eta=0.5$ and 0.95 .

where

$$
\widehat{\mathbf{u}}=\widehat{u} \mathbf{i}+\widehat{v} \mathbf{j}, \quad \mathbf{U}=U \mathbf{i}+V \mathbf{j} .
$$

The discretization parallels that performed for the base flow and leads to the following generalized eigenvalue problem: ${ }^{5}$

$$
\mathbf{G x}=\sigma \mathbf{B x},
$$

where $\sigma=f\left(\operatorname{Re}, k, e^{*}, \eta\right)$. The vector $\mathbf{x}$ is comprised of the unknown vclocity and pressure coefficients, and $\mathbf{B}$ is a square diagonal matrix whose entries corresponding to the continuity equation and the homogeneous boundary conditions are zero. The zeros in the matrix $B$ result in "infinite" eigenvalues that preclude the use of a standard power routine to isolate the most dangerous eigenvalue (i.e., the eigenvalue with the largest real part). ${ }^{23,24}$ We therefore choose to solve the eigenvalue problem directly with the EISPACK routine RGG. Since the base flow is Reynolds number dependent, the critical parameters $\operatorname{Re}_{c}$ and $k_{c}$ must be determined in an iterative fashion. Note that $\operatorname{Re}_{c}$ and $k_{c}$ correspond to the real part of $\sigma$ being equal to zero.

We tested our code by confirming the critical parameters found by Oikawa et al. ${ }^{11}$ for eccentric circular cylinders, including an instance of a Hopf bifurcation. ${ }^{5}$ To avoid degradation of spectral accuracy due to the sharp corners in the elliptical cylinder problem, we divide the flow do-

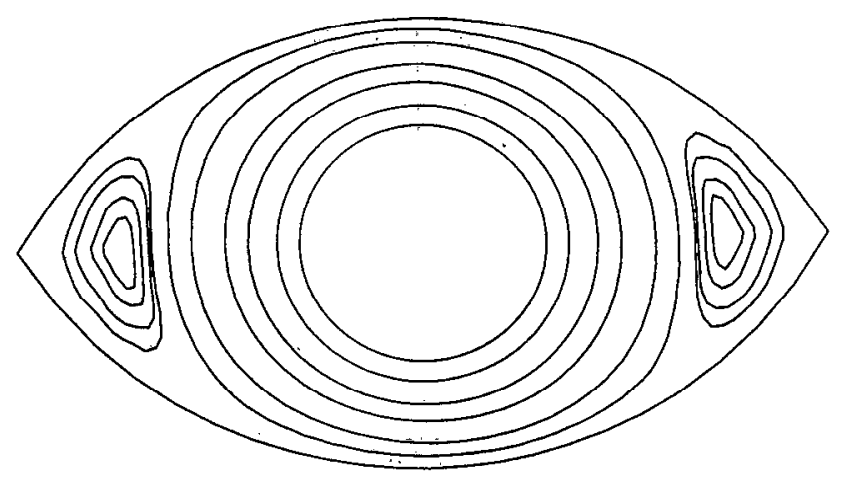

FIG. 3. Streamlines for base flow. $\operatorname{Re}=51.75, \eta=0.5, e^{*}=2$. 


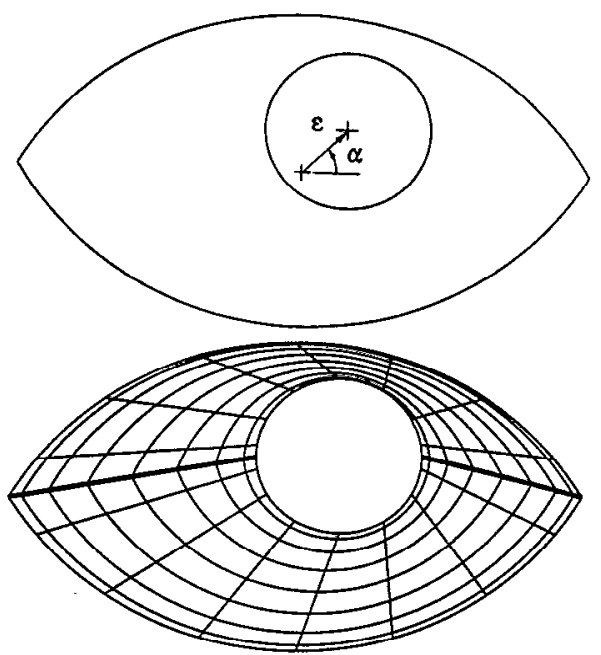

\begin{tabular}{|c|c|c|c|}
\hline$\alpha$ & $\epsilon$ & $R e_{c}$ & $k_{c}$ \\
\hline- & 0 & 51.7 & 2.30 \\
0 & 0.5 & 54.5 & 2.40 \\
$\pi / 2$ & 0.5 & 73.7 & 2.81 \\
\hline
\end{tabular}

FIG. 4. Geometry, typical grid $(N=8)$, and critical parameters for eccentric elliptical cylinders. $\eta=0.5, e^{*}=2$.

main into two elements such that the element corners coincide with the outer cylinder corners.

Figure 2 shows how the critical Reynolds number and wave number change with ellipticity $e^{*}$. We show results for two minimum gap radius ratios: $\eta=0.5$ and $\eta=0.95$. For all the $\eta=0.5$ runs, we used a square truncation with $N=10$ in each of the two elements. For $\eta=0.95$, we used $N=10$ for $e^{*}=0.1$ and 0.5 , and $N=14$ for $e^{*}=1$ and 2 . We ensured convergence of the eigenvalues by confirming the eigenvalues with higher truncations.

Figure 3 shows streamlines for the base flow with $e^{*}=2$ and $\eta=0.5$ at a slightly supercritical state where $\operatorname{Re}=51.75$. The truncation is $N=10$. Analysis showed that the slight irregularities in the illustrated corner vortices are due not to poor resolution of the base flow velocity solution, but to limitations in our contour-plotting routine.

As the inner cylinder is moved from the concentric position, it might be expected that the critical Reynolds number and wave number would increase. This would be consistent with the results for eccentric circular cylinders from Oikawa et al. ${ }^{11}$ With eccentric elliptical cylinders, two additional parameters are introduced: the eccentricity ratio $\epsilon$ and the angle $\alpha$, as seen in Fig. 4 . The eccentricity ratio is defined as $\epsilon=\hat{e} / d$, where $\hat{e}$ is the distance between the inner and outer cylinder centers. Note that, for any angles other than $\alpha= \pm \pi / 2, \epsilon$ can exceed unity. In Fig. 4, we tabulate three cases: $\epsilon=0 ; \epsilon=0.5, \alpha=\pi / 2$; and $\epsilon=0.5$, $\alpha=0$. As expected, the critical parameters for both eccentric cases are higher than those for the concentric case. For a given eccentricity, the flow is more stable for angles that result in smaller clearances.

In conclusion, we find that increasing ellipticity destabilizes the flow. It is well known that, for the concentric cylinder problem, wider gaps are associated with lower critical Reynolds numbers. Since the appearance of Taylor vortices may be dictated by an average gap in the elliptical cylinder case, the decreasing critical Reynolds number with increasing ellipticity makes sense. This is, however, in contrast to Snyder's ${ }^{15}$ experimental observation that, for a spinning inner circular cylinder and stationary outer square cylinder arrangement, the appearance of vortices was dictated by the minimum gap.

Increasing ellipticity widens the Taylor vortices in the $z$ direction. Snyder noted that the wavelength of the vortices in his geometry lay somewhere between twice the minimum and maximum gap widths. We find the same phenomena here, as is evident by the increasing ellipticity/ decreasing wave-number relationship shown in Fig. 2.

As with eccentric circular cylinders, increasing eccentricity stabilizes the flow and results in smaller vortices. For given eccentricity, ellipticity, and $\eta$, the flow is more stable for angles that result in smaller clearances between the two cylinders.

We found no Hopf bifurcation for the elliptical geometry results presented here. However, since Hopf bifurcations do occur for eccentric cylinders with small gap and high eccentricity, ${ }^{11}$ we suspect that at large enough values of $\eta$ and $e^{*}$ a Hopf bifurcation will also occur for elliptical gcomctries. Computer resource limitations prevented us from trying the necessary higher truncations. Perhaps a mapping that stretches the radial coordinate in the narrowest parts of the flow field would allow solutions with reasonable truncations.

\section{ACKNOWLEDGMENT}

This work was supported by National Science Foundation Contract No. DMC-8716766

${ }^{2)}$ Present address: University of Detroit Mercy, Detroit, Michigan 48221. ${ }^{1}$ O. Pinkus, "Analysis of elliptical bearings," Trans. ASME 78, 965, (1956).

${ }^{2}$ O. Pinkus, "Power loss in elliptical and 3-lobe bearings," Trans. ASME 78, 899 (1956).

${ }^{3}$ A. Kumar, R. Sinhasan, and D. V. Singh, "Performance characteristics of two-lobe hydrodynamic journal bearings," J. Lubr. Technol. 102, 425 (1980).

${ }^{4} \mathrm{O}$. Reynolds, "On the theory of lubrication and its application to $\mathrm{Mr}$. Beauchamp tower's experiments," Philos. Trans. R. Soc. 177, 157 (1886).

${ }^{5} \mathrm{M}$. R. Schumack, "A spectral domain decomposition method with applications to flows between cylinders," Ph.D. thesis, Univcrsity of Michigan, 1991 (unpublished).

${ }^{6}$ Tribology Handbook, edited by M. J. Neale (Butterworths, London, 1973).

${ }^{7}$ R. C. DiPrima and H. L. Swinney, "Instabilities and transition in flow between concentric rotating cylinders," in Hydrodynamic Instabilities and the Transition to Turbulence, edited by H. L. Swinney and J. P. Gollub (Springer-Verlag, Berlin, 1985).

${ }^{8}$ R. C. DiPrima and J. T. Stuart, "Non-local effects in the stability of flow between eccentric rotating cylinders," J. Fluid Mech. 54, 393 (1972).

${ }^{9}$ R. C. DiPrima and J. T. Stuart, "The nonlinear calculation of Taylorvortex flow between eccentric rotating cylinders," J. Fluid Mech. 67, 85 (1975).

${ }^{10}$ P. M. Eagles, J. T. Stuart, and R. C. DiPrima, "The effects of eccentricity on torque and load in Taylor-vortex flow," J. Fluid Mech. 87, 209 (1978).

${ }^{11}$ M. Oikawa, T. Karasudani, and M. Funakoshi, "Stability of flow between eccentric rotating cylinders," J. Phys. Soc. Jpn. 58, 2355 (1989).

${ }^{12} \mathrm{~J}$. H. Vohr, "An experimental study of Taylor vortices and turbulence 
in flow between eccentric rotating cylinders," J. Lubr. Technol. 90, 285 (1968).

${ }^{13}$ T. Karasudani, "Non-axis-symmetric Taylor vortex flow in eccentric rotating cylinders," J. Phys. Soc. Jpn. 56, 855 (1987).

${ }^{14} \mathrm{E}$. Lewis, "Steady flow between a rotating circular cylinder and fixed square cylinder," J. Fluid Mech. 95, 497 (1979).

${ }^{15} \mathrm{H}$. A. Snyder, "Experiments on rotating flows between noncircular cylinders," Phys. Fluids 11, 1606 (1968).

${ }^{16} \mathrm{~T}$. Mullin and A. Lorenzen, "Bifurcation phenomena in flows between a rotating circular cylinder and a stationary square outer cylinder," J. Fluid Mech. 157, 289 (1985).

${ }^{17} \mathrm{~T}$. Terada and $\mathrm{K}$. Hattori, "Formation of vortices by rotating disc, sphere, or cylinder," Rep. Tokyo Univ. Aeronaut. Res. Inst. 2, 287 (1926).

${ }^{18}$ E. M. Ronquist, "Legendre spectral element methods for the imcompressible Navier-Stokes equations," Ph.D. thesis, Massachusetts Institute of Technology, 1988 (unpublished).
${ }^{19}$ Y. Maday and A. T. Patera, "Spectral element methods for the imcompressible Navier-Stokes equations," in State-of-the-Art Surveys in Computational Mechanics, edited by A. K. Noor (ASME, New York, 1988).

${ }^{20} \mathrm{G}$. E. Karniadakis, "Spectral element simulations of laminar and turbulent flows in complex geometries," App. Numer. Math. 6, 85 (1989/ 90).

${ }^{21}$ T. A. Zang, "On the rotation and skew-symmetric forms for imcompressible flow simulations," Appl. Numer. Math. 7, 27 (1991).

${ }^{22}$ M. R. Schumack, W. W. Schultz, and J. P. Boyd, "Spectral method solution of the Stokes equations on nonstaggered grids," J. Comput. Phys. 94, 30 (1991).

${ }^{23}$ D. A. Goussis and A. J. Pearlstein, "Removal of infinite eigenvalues in the generalized matrix eigenvalue problem," J. Comput. Phys. 84, 242 (1989).

${ }^{24} \mathrm{~K}$. N. Christodoulou and L. E. Scriven, "Finding leading modes of a viscous free surface flow: An asymmetric generalized eigenproblem," J. Sci. Comput. 3, 355 (1988). 